\title{
The Influence of Gastroesophageal Reflux Disease on Daytime Sleepiness and Depressive Symptom in Patients With Obstructive Sleep Apnea
}

\author{
Hyeon Hui Kang, ${ }^{1}$ Chul-Hyun Lim, ${ }^{2 *}$ Jung Hwan $\mathrm{Oh}^{2}{ }^{2}$ Min-Jae Cho, ${ }^{2}$ and Sang Haak Lee ${ }^{2}$ \\ ${ }^{I}$ Department of Internal Medicine, Ulsan University Hospital, University of Ulsan College of Medicine, Ulsan, Korea and ${ }^{2}$ Department of Internal \\ Medicine, College of Medicine, The Catholic University of Korea, Seoul, Korea
}

\section{Background/Aims}

As there is insufficient evidence for a relationship between gastroesophageal reflux disease (GERD) and obstructive sleep apnea (OSA), we investigated whether OSA diagnosed by polysomnography (PSG) is related to GERD.

\section{Methods}

A total of 402 subjects was evaluated. Overnight PSG was performed and a few questionnaires on GERD, anxiety, depression, and daytime sleepiness were administered. An apnea-hypopnea index $<5$ was the classification criterion for subjects without OSA. Subjects with heartburn or acid regurgitation at least once a week were classified as having GERD.

\section{Results}

Among the 402 subjects, 318 had OSA and 84 did not. The prevalence of GERD was $12.9 \%$ among patients with OSA and $10.7 \%$ among those without $(P=0.590)$. The prevalence of GERD did not correlate with OSA severity $(P=0.474)$. Patients with OSA with GERD had higher Stanford Sleepiness Scale $(P=0.004)$, Epworth Sleepiness Scale $(P=0.001)$, and depression $(P<0.001)$ scores than patients with OSA without GERD. Subjects with nocturnal gastroesophageal reflux symptoms had a higher body mass index, waist-to-height ratio, and waist circumference-to-height index than those without symptoms. Multiple logistic regression showed that higher Epworth Sleepiness Scale and depression scores were independent factors associated with GERD in patients with OSA.

\section{Conclusions}

The prevalence of GERD in patients with OSA was $12.9 \%$. The prevalence of GERD did not correlate with OSA severity. Daytime sleepiness and depression seem to be associated with GERD in patients with OSA, while nocturnal reflux symptoms seem to be related to obesity in OSA.

(J Neurogastroenterol Motil 2021;27:215-222)

\section{Key Words}

Depression; Gastroesophageal reflux; Obesity; Polysomnography; Sleep apnea, obstructive

\section{Received: April 10, 2020 Revised: June 1, 2020 Accepted: June 15, 2020}

(a) This is an Open Access article distributed under the terms of the Creative Commons Attribution Non-Commercial License (http://creativecommons. org/licenses/by-nc/4.0) which permits unrestricted non-commercial use, distribution, and reproduction in any medium, provided the original work is properly cited.

*Correspondence: Chul-Hyun Lim, MD, PhD Division of Gastroenterology, Department of Internal Medicine, Eunpyeong St. Mary's Hospital, College of Medicine, The Catholic University of Korea, 1021, Tongil-ro, Eunpyeong-gu, Seoul 03312, Korea Tel: +82-2-2030-4316, Fax: +82-2-2030-4641, E-mail: diluck@catholic.ac.kr 


\section{Introduction}

The prevalence of gastroesophageal reflux disease (GERD) has been gradually increasing in the Eastern world. The prevalence of symptom-based GERD was reported at 5.2-8.5\% from 2005 to 2010 in Eastern Asia. ${ }^{1}$ During sleep, esophageal and gastric motility is decreased, and the time for esophageal mucosa exposure to gastric acid is increased. This may exacerbate the symptoms of gastroesophageal reflux, which causes awakening and thus interrupts sleep. GERD and obstructive sleep apnea (OSA) which is one of the most common sleep disorders, are thought to affect each other, but the relationship has never been clearly demonstrated. There is some evidence for a link between GERD and OSA. ${ }^{2}$ Omeprazole therapy for GERD has been shown to reduce the occurrence of apnea attacks. ${ }^{3}$ The use of proton pump inhibitors can improve the quality of life associated with sleep disturbance. ${ }^{4}$ In the case-control study, it has been suggested that OSA may increase the risk of Barrett's esophagus through obesity and GERD.' The known risk factors for OSA are advanced age, male sex, and obesity. ${ }^{6}$ Furthermore, some risk factors are common for both disorders. Obesity, for example, is a major risk factor for OSA and GERD. Organization for Economic Cooperation and Development statistics show that obesity rates range from $4.2 \%$ (Japan) to $40.0 \%$ (USA) of the total adult population when a body mass index (BMI) of $30 \mathrm{~kg} / \mathrm{m}^{2}$ or more is used as the defining criterion for obesity. ${ }^{7}$ Over the years, the prevalence of overweight and obesity has also increased in Korea.

While weight loss has been shown to result in improvements in OSA parameters, ${ }^{8}$ it is unclear whether it is strongly associated with both diseases because of similar risk factors, or whether there might be a causal relationship between both OSA and GERD. Furthermore, it is not clear whether OSA severity has any causal relationship with reflux symptoms. The aim of this study is thus to investigate the prevalence of GERD in patients with OSA diagnosed by overnight polysomnography (PSG). In addition, we assessed the risk factors for GERD in patient with OSA.

\section{Materials and Methods}

\section{Subjects}

We consecutively enrolled 424 subjects who were suspected of having OSA and underwent full overnight PSG at the sleep clinic of St. Paul's Hospital, the Catholic University of Korea, between September 2009 and September 2015. Twenty-two subjects who

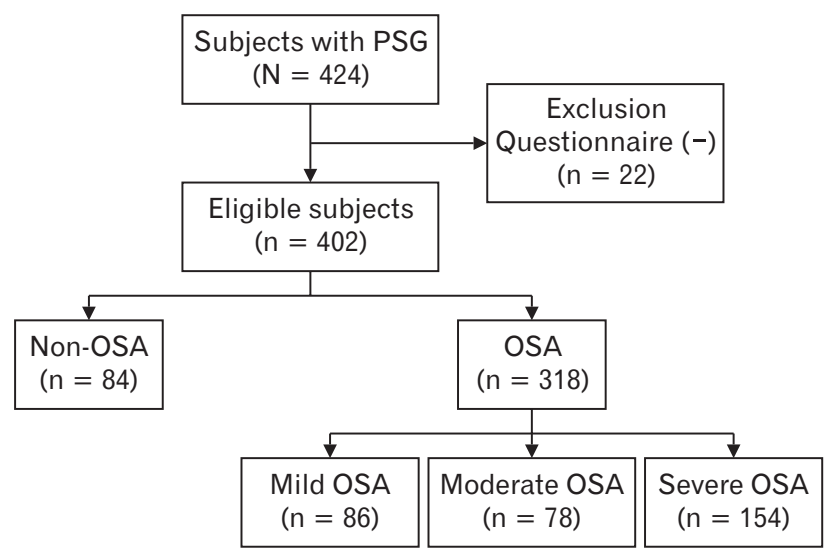

Figure 1. Flow chart of the study. PSG, overnight polysomnography; OSA, obstructive sleep apnea.

did not complete the questionnaire were excluded. The subjects completed several questionnaires on reflux symptoms, anxiety and depression, and daytime sleepiness. Finally, 402 subjects were included in this study. The study, summarized in Figure 1, was approved by the institutional review board of our hospital (IRB No. PC14RISI0037).

\section{Polysomnography}

PSG (Somnostar Pro 7-3a; Somnostar/Cardinal Health, Dublin, OH, USA) was conducted overnight. Apnea was defined as a reduction in airflow of more than $90.0 \%$ lasting at least 10 seconds. Hypopnea was defined as a decrease in airflow of more than $50.0 \%$ for at least 10 seconds and a decrease in arterial oxygen saturation of more than $4.0 \%$ or arousal. ${ }^{9}$ The apnea-hypopnea index (AHI) was defined as the sum of apneas and hypopneas per hour. OSA severity was defined as mild for an $\mathrm{AHI} \geq 5$ and $<15$, moderate for an $\mathrm{AHI} \geq 15$ and $\leq 30$, and severe for an AHI $>30$ /hour. Total sleep time was defined as the total amount of recorded sleep during the PSG. The sleep period time was defined as the elapsed time from sleep onset to the end of the last epoch of sleep. Sleep efficiency was defined as the percentage of time spent asleep while in bed. Sleep is broken down into 5 phases: wake, N1, N2, N3, and R. Stages N1 to N3 are considered non-rapid eye movement sleep, each progressively going deeper. Stage $\mathrm{R}$ is referred to as rapid eye movement sleep.

\section{Questionnaires}

\section{Reflux questionnaire}

The reflux questionnaire was based on a questionnaire that our 
group has previously used. ${ }^{10-14}$ The subjects were asked questions about the occurrence of typical reflux symptoms such as heartburn and acid regurgitation and answered as follows: 0 , none; 1 , less than once a month; 2 , approximately once a month; 3 , approximately once a week; 4, more than twice a week; and 5, every day. Subjects with heartburn or acid regurgitation at least once a week were designated as having GERD. Subjects were asked about the presence of nocturnal reflux symptoms if they had woken up while sleeping due to reflux symptoms. ${ }^{14}$

\section{Anxiety and depression score}

As the questionnaire on anxiety and depression, we used the Korean version of the Hospital Anxiety and Depression Scale, which has been validated. ${ }^{15,16}$ Scores of 8 or higher were defined as abnormal states of anxiety and depression.

\section{Other questionnaires}

Two questionnaires were used to identify daytime sleepiness. The Stanford Sleepiness Scale (SSS) was used to assess weekly awakening, classified into 1 to 7 points according to the degree of alertness. ${ }^{17}$ The Epworth Sleepiness Scale (ESS) was used to assess weekly sleep overload in 8 situations where sleep occurs in everyday life. If a patient's total score on the ESS was higher than 10, he/she was classified as having persistent drowsiness. ${ }^{18,19}$

\section{Obesity-related Parameters}

We used the BMI, the waist-to-height ratio (WHR), and the waist circumference-to-height index (WHI, waist circumference) height ${ }^{2}$ in $\mathrm{cm} / \mathrm{m}^{2}$ ) as tools to detect obesity. The WHR has been shown to be superior to the BMI and waist circumference for identifying cardiometabolic risk factors in both men and women, ${ }^{20}$ while

Table 1. Demographics of the Study Subjects

\begin{tabular}{|c|c|c|c|c|}
\hline Variables & OSA $(n=318)$ & Non-OSA $(\mathrm{n}=84)$ & Total $(\mathrm{N}=402)$ & $P$-value \\
\hline Age (yr) & $50.9 \pm 12.6$ & $45.7 \pm 17.8$ & $49.8 \pm 14.0$ & $0.016^{\mathrm{a}}$ \\
\hline Sex & & & & $<0.001^{\mathrm{a}}$ \\
\hline Men & $237(74.5)$ & $34(40.5)$ & $271(67.4)$ & \\
\hline Women & $81(25.5)$ & $50(59.5)$ & $131(32.6)$ & \\
\hline Hypertension & $105(33.0)$ & $19(22.6)$ & $124(30.9)$ & 0.066 \\
\hline $\mathrm{DM}$ & $43(13.5)$ & $11(13.1)$ & $54(13.4)$ & 0.919 \\
\hline Alcohol & $195(61.5)$ & $40(47.6)$ & $235(58.6)$ & $0.022^{\mathrm{a}}$ \\
\hline Smoking & & & & $<0.001^{\mathrm{a}}$ \\
\hline Never & $161(50.6)$ & $66(78.6)$ & $227(56.5)$ & \\
\hline Current & $62(19.5)$ & $6(7.1)$ & $68(16.9)$ & \\
\hline Ex-smoker & $95(29.9)$ & $12(14.3)$ & $107(26.6)$ & \\
\hline Aspirin & $53(16.7)$ & $9(10.7)$ & $62(15.5)$ & 0.176 \\
\hline NSAIDs & $22(6.9)$ & $7(1.8)$ & $29(7.2)$ & 0.661 \\
\hline Anti-anxiety drug & $20(6.3)$ & $8(9.5)$ & $28(7.0)$ & 0.304 \\
\hline PPI & $29(9.2)$ & $8(9.5)$ & $37(9.2)$ & 0.916 \\
\hline GERD & $41(12.9)$ & $9(10.7)$ & $50(12.4)$ & 0.591 \\
\hline $\operatorname{BMI}\left(\mathrm{kg} / \mathrm{m}^{2}\right)$ & $27.2 \pm 4.3$ & $23.3 \pm 3.3$ & $26.4 \pm 4.4$ & $<0.001^{\mathrm{a}}$ \\
\hline WHR & $0.57 \pm 0.06$ & $0.52 \pm 0.06$ & $0.56 \pm 0.07$ & $<0.001^{\mathrm{a}}$ \\
\hline $\mathrm{WHI}\left(\mathrm{cm} / \mathrm{m}^{2}\right)$ & $34.36 \pm 4.68$ & $32.03 \pm 4.82$ & $33.87 \pm 4.80$ & $<0.001^{\mathrm{a}}$ \\
\hline SSS score & $2.55 \pm 1.07$ & $2.63 \pm 0.92$ & $2.56 \pm 1.04$ & 0.238 \\
\hline ESS score & $9.49 \pm 4.07$ & $8.62 \pm 3.98$ & $9.31 \pm 4.06$ & 0.067 \\
\hline \multicolumn{5}{|l|}{ HADS } \\
\hline Anxiety score & $4.69 \pm 3.75$ & $6.43 \pm 3.87$ & $5.06 \pm 3.84$ & $<0.001^{\mathrm{a}}$ \\
\hline Depression score & $5.99 \pm 3.65$ & $7.58 \pm 4.29$ & $6.32 \pm 3.84$ & $0.002^{\mathrm{a}}$ \\
\hline AHI (events/hr) & $35.45 \pm 25.28$ & $2.09 \pm 1.44$ & $28.48 \pm 26.27$ & $<0.001^{\mathrm{a}}$ \\
\hline
\end{tabular}

${ }^{\mathrm{a}} \mathrm{P}<0.05$ : statistical significance.

OSA, obstructive sleep apnea; DM, diabetes mellitus; PPI, proton pump inhibitor; GERD, gastroesophageal reflux disease; BMI, body mass index; WHR, waistto-height ratio; WHI, waist circumference-to-height index; SSS, Stanford Sleepiness Scale; ESS, Epworth Sleepiness Scale; HADS, Hospital Anxiety and Depression Scale; AHI, apnea-hypopnea index.

Data are presented as mean $\pm \mathrm{SD}$ or $\mathrm{n}(\%)$. 
the WHI is a new index of abdominal obesity based on height in addition to waist circumference. ${ }^{21}$

\section{Statistical Methods}

The patient's categorical data are presented as mean \pm standard deviation. Categorical variables were evaluated with chi-square tests or Fisher's exact test. Continuous variables were evaluated using $t$ tests. Differences between GERD and controls were assessed using chi-square and $t$ tests. Both univariate and multivariate analyses were performed to identify risk factors for GERD, with SAS version 9.4 (SAS Institute Inc, Cary, NC, USA). The level of statistical significance was $P<0.05$ for all analyses.

Table 2. Clinical Characteristics of the Subjects According to Gastroesophageal Reflux Disease

\begin{tabular}{lccc}
\hline \multirow{2}{*}{ Variables } & \multicolumn{2}{c}{ OSA $(\mathrm{N}=318)$} & \\
\cline { 2 - 3 } & GERD $(+)$ & GERD $(-)$ & P-value \\
& $(\mathrm{n}=41)$ & $(\mathrm{n}=277)$ & \\
\hline Age $(\mathrm{yr})$ & $52.1 \pm 11.2$ & $50.7 \pm 12.8$ & 0.518 \\
Sex & & & 0.831 \\
Men & $30(73.2)$ & $207(74.7)$ & \\
Women & $11(26.8)$ & $70(25.3)$ & \\
Hypertension & $13(31.71)$ & $92(33.2)$ & 0.848 \\
DM & $8(19.51)$ & $35(12.6)$ & 0.229 \\
Alcohol & $22(53.0)$ & $173(62.5)$ & 0.365 \\
Smoking & & & 0.703 \\
Never & $22(53.7)$ & $139(50.2)$ & \\
Current & $9(22.0)$ & $53(19.1)$ & \\
Ex-smoker & $10(24.4)$ & $85(30.7)$ & \\
Aspirin & $4(9.8)$ & $49(17.8)$ & 0.200 \\
NSAIDs & $2(4.9)$ & $20(6.3)$ & 0.751 \\
Anti-anxiety drug & $3(7.3)$ & $17(6.2)$ & 0.732 \\
PPI & $9(22.0)$ & $20(7.3)$ & $0.006^{\mathrm{a}}$ \\
BMI $\left(\mathrm{kg} / \mathrm{m}^{2}\right)$ & $28.1 \pm 4.4$ & $27.0 \pm 4.3$ & 0.117 \\
WHR & $0.58 \pm 0.07$ & $0.57 \pm 0.06$ & 0.128 \\
WHI $\left(\mathrm{cm} / \mathrm{m}^{2}\right)$ & $35.52 \pm 5.12$ & $34.19 \pm 4.60$ & 0.102 \\
SSS score & $3.00 \pm 1.22$ & $2.48 \pm 1.03$ & $0.004^{\mathrm{a}}$ \\
ESS score & $11.71 \pm 4.91$ & $9.16 \pm 3.83$ & $0.001^{\mathrm{a}}$ \\
HADS & & & \\
Anxiety score & $5.20 \pm 4.00$ & $4.62 \pm 3.72$ & 0.454 \\
Depression score & $8.05 \pm 3.75$ & $5.68 \pm 3.54$ & $<0.001^{\mathrm{a}}$ \\
\hline
\end{tabular}

${ }^{a} P<0.05$ : statistical significance.

OSA, obstructive sleep apnea; GERD, gastroesophageal reflux disease; DM, diabetes mellitus; PPI, proton pump inhibitor; BMI, body mass index; WHR, waist-to-height ratio; WHI, waist circumference-to-height index; SSS, Stanford Sleepiness Scale; ESS, Epworth Sleepiness Scale; HADS, Hospital Anxiety and Depression Scale.

Data are presented as mean $\pm \mathrm{SD}$ or $\mathrm{n}(\%)$.

\section{Results}

\section{Baseline Characteristics}

Among the 402 subjects who all underwent PSG, 318 (79.1\%) had OSA. Of these patients, $74.5 \%$ were men $(n=237)$ and $25.5 \%(\mathrm{n}=81)$ were women. Table 1 shows the clinical characteristics according to the presence of OSA. Fifty subjects (12.4\%) had reflux symptoms at least once a week. Forty-one patients with OSA (12.9\%) and 9 patients in the non-OSA group (10.7\%) had reflux symptoms, with the difference not being statistically significant $(P=0.591)$. Table 2 shows the clinical characteristics of the OSA according to the presence of GERD. Subjects with OSA with GERD had higher scores on the SSS than subjects with OSA without GERD $(3.0 \pm 1.2$ vs $2.5 \pm 1.0, P=0.004)$. ESS scores were also much higher in patients with GERD than in patients without GERD $(11.7 \pm 4.9$ vs $9.2 \pm 3.8, P=0.001)$. Depression scores were higher in patients with GERD than in patients without GERD $(8.1 \pm 3.8$ vs $5.7 \pm 3.5, P<0.001)$. The prevalence of GERD did not correlate with OSA severity $(P=0.608)$ (Fig. 2).

\section{Obesity-related Parameters in Gastroesophageal Reflux Disease With Obstructive Sleep Apnea}

Patients with GERD had a higher BMI, WHI, and WHR than patients without GERD, there was no statistically significant difference. However, if the patient was classified based only on the nocturnal reflux symptoms regardless of GERD definition or other GERD symptoms, patients with OSA with nocturnal symptoms had a higher BMI, WHI, and WHR than patients with OSA

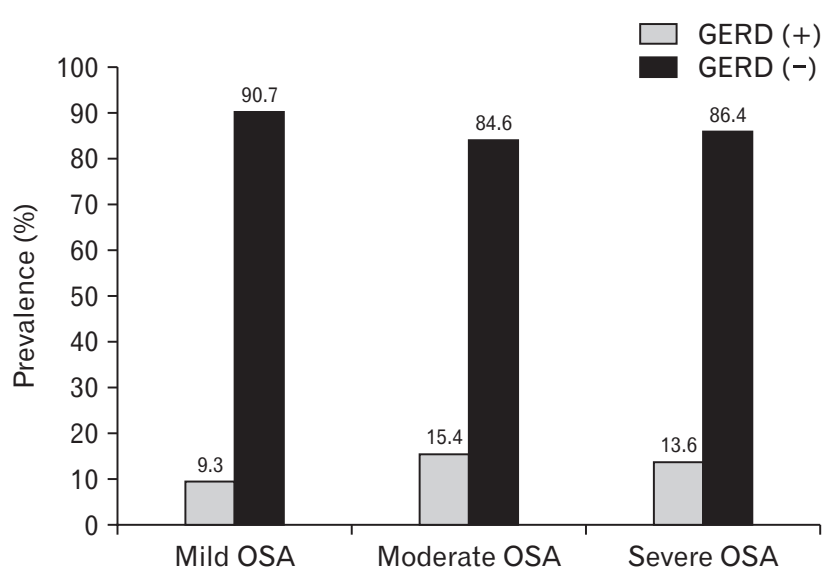

Figure 2. The prevalence of gastroesophageal reflux disease (GERD) according to obstructive sleep apnea (OSA) severity. 
Table 3. Obesity-related Parameters According to the Presence of Nocturnal Reflux Symptoms in Patients With Obstructive Sleep Apnea

\begin{tabular}{|c|c|c|c|c|}
\hline \multirow{3}{*}{ Variables } & \multicolumn{2}{|c|}{ Nocturnal reflux symptoms } & \multirow{3}{*}{$\begin{array}{c}\text { Total } \\
(\mathrm{N}=318)\end{array}$} & \multirow{3}{*}{$P$-value } \\
\hline & $(+)$ & $(-)$ & & \\
\hline & $(\mathrm{n}=52)$ & $(n=266)$ & & \\
\hline GERD & $18(34.6)$ & $23(8.7)$ & $41(12.9)$ & $<0.001^{\mathrm{b}}$ \\
\hline $\operatorname{BMI}\left(\mathrm{kg} / \mathrm{m}^{2}\right)$ & $28.2 \pm 4.1$ & $26.9 \pm 4.3$ & $27.2 \pm 4.3$ & $0.031^{\mathrm{b}}$ \\
\hline WHR & $0.59 \pm 0.06$ & $0.57 \pm 0.06$ & $0.57 \pm 0.06$ & $0.008^{\mathrm{b}}$ \\
\hline WHI & $35.48 \pm 4.77$ & $34.14 \pm 4.65$ & $34.36 \pm 4.68$ & $0.047^{\mathrm{b}}$ \\
\hline
\end{tabular}

${ }^{a}$ Nocturnal reflux symptoms was classified based only on the nocturnal reflux symptoms regardless of gastroesophageal reflux disease (GERD) definition or other GERD symptoms.

${ }^{\mathrm{b}} P<0.05$ : statistical significance.

BMI, body mass index; WHR, waist-to-height ratio; WHI, waist circumference-to-height index.

Data are presented as $\mathrm{n}(\%)$ or mean $\pm \mathrm{SD}$.

Table 4. Risk Factors for Gastroesophageal Reflux Disease Among Obstructive Sleep Apnea Based on Logistic Regression Analyses

\begin{tabular}{|c|c|c|c|c|}
\hline \multirow[b]{2}{*}{ Variables } & \multicolumn{2}{|c|}{ Univariate analysis } & \multicolumn{2}{|c|}{ Multivariate analysis } \\
\hline & $\begin{array}{c}\text { OR } \\
(95 \% \mathrm{CI})\end{array}$ & $P$-value & $\begin{array}{c}\text { OR } \\
(95 \% \mathrm{CI})\end{array}$ & $P$-value \\
\hline SSS score & $\begin{array}{c}1.47 \\
(1.12-1.92)\end{array}$ & $0.005^{\mathrm{a}}$ & $\begin{array}{c}1.29 \\
(0.95-1.75)\end{array}$ & 0.100 \\
\hline ESS score & $\begin{array}{c}1.15 \\
(1.07-1.25)\end{array}$ & $<0.001^{\mathrm{a}}$ & $\begin{array}{c}1.12 \\
(1.03-1.22)\end{array}$ & $0.008^{\mathrm{a}}$ \\
\hline Depression score & $\begin{array}{c}1.18 \\
(1.08-1.29)\end{array}$ & $<0.001^{\mathrm{a}}$ & $\begin{array}{c}1.16 \\
(1.06-1.27)\end{array}$ & $0.001^{\mathrm{a}}$ \\
\hline
\end{tabular}

${ }^{a} P<0.05$ : statistical significance.

SSS, Stanford Sleepiness Scale; ESS, Epworth Sleepiness Scale.

without nocturnal symptoms (Table 3).

\section{Risk Factors for Gastroesophageal Reflux Disease in Patients With Obstructive Sleep Apnea}

High scores of SSS, ESS, and depression were statistically significant risk factors for GERD in OSA by univariate logistic regression. Multivariate logistic regression then identified high ESS and depression scores as risk factors for GERD in OSA (Table 4).

\section{Polysomnography Findings According to Gastroesophageal Reflux Disease Symptoms}

There was no statistically significant difference compared to patients without GERD in study groups (Table 5).
Table 5. Polysomnographic Findings of the Study Groups According to the Presence of Gastroesophageal Reflux Disease

\begin{tabular}{|c|c|c|c|}
\hline \multirow[b]{2}{*}{ Variables } & \multicolumn{2}{|c|}{ OSA $(\mathrm{N}=318)$} & \multirow[b]{2}{*}{$P$-value } \\
\hline & $\begin{array}{c}\operatorname{GERD}(+) \\
(\mathrm{n}=41)\end{array}$ & $\begin{array}{l}\text { GERD (-) } \\
(\mathrm{n}=277)\end{array}$ & \\
\hline Minimum oxygen saturation (\%) & $77.7 \pm 10.5$ & $79.2 \pm 8.6$ & 0.567 \\
\hline Sleep period time $(\min )$ & $396.1 \pm 52.4$ & $405.9 \pm 57.7$ & 0.429 \\
\hline Total sleep time (min) & $333.3 \pm 77.0$ & $339.4 \pm 65.6$ & 0.826 \\
\hline Sleep efficiency (\%) & $81.1 \pm 16.2$ & $81.9 \pm 13.4$ & 0.814 \\
\hline $\mathrm{N} 1(\%)$ & $35.6 \pm 18.9$ & $33.9 \pm 16.0$ & 0.650 \\
\hline $\mathrm{N} 2(\%)$ & $44.6 \pm 13.1$ & $45.1 \pm 12.5$ & 0.947 \\
\hline N3 (\%) & $5.0 \pm 6.8$ & $5.2 \pm 8.3$ & 0.788 \\
\hline Arousal index & $24.9 \pm 28.0$ & $21.3 \pm 23.2$ & 0.707 \\
\hline AHI (events/hr) & $40.8 \pm 30.6$ & $34.7 \pm 24.4$ & 0.376 \\
\hline
\end{tabular}

OSA, obstructive sleep apnea; GERD, gastroesophageal reflux disease; AHI, apnea-hypopnea index.

Data are presented as mean $\pm \mathrm{SD}$.

\section{Discussion}

This study revealed that the prevalence of GERD in patients with OSA was $12.9 \%$. The prevalence of GERD did not correlate with OSA severity. We also found that daytime sleepiness and depression seem to be associated with GERD and nocturnal reflux symptom may be related to obesity in patients with OSA.

A study conducted in the USA showed that the severity of OSA does not correlate with the percentage of patients with GERD. ${ }^{22}$ Another study has also failed to demonstrate that more severe OSA is associated with worse GERD, ${ }^{23}$ with the authors suggesting that both diseases are common entities that share similar risk factors but are not causally linked. Contrary to these studies, a study in a Korean sample recently found that reflux esophagitis was associated with more severe OSA. ${ }^{24}$ In 216 patients who underwent endoscopy and PSG, OSA parameters including AHI were found to be worse in the reflux esophagitis than in the no esophagitis group. In our study, about $75.0 \%$ of the subjects who underwent full overnight PSG had OSA, and $12.5 \%$ had of these GERD, similar to another study conducted in Korea, where $9.0 \%$ of patients who underwent PSG were found to have GERD ${ }^{25}$ A recent study with 1104 patients conducted in Turkey reported that $38.9 \%$ had GERD (once a week of heartburn and/or regurgitation) in the OSA group, showing a higher prevalence of GERD with OSA compared with our study. However, non-OSAS group also had a prevalence of $32.0 \%$, which is not much different from the OSA group. Similar to our results, this study suggested that the prevalence of GERD between OSA and non-OSAS was not different 
regardless of the different prevalence of GERD in the population. ${ }^{26}$ One explanation for this difference might be differences in BMI: the low prevalence of GERD in our study might be related to the fact that our patient population was less obese than usual samples in other countries (mean BMI: $27.2 \mathrm{~kg} / \mathrm{m}^{2}$ ). Even though the prevalence of GERD is increasing in Asia, it is still higher in Western countries. In the above-mentioned study conducted in the USA, for example, GERD was found in $22.0 \%$ of 1023 subjects. ${ }^{22}$

In this study, obesity-related parameters including BMI, WHR, and WHI did not differ between in OSA patient with and without GERD. However, the obesity-related parameters were found to be higher in patients with nocturnal reflux symptoms. This suggests that obesity may be associated with nocturnal reflux symptoms. WHI, a new index of abdominal obesity, could be a more accurate risk factor for GERD than the BMI, since GERD seems to be more associated with abdominal obesity than with overall body fat. Another study also suggested that abdominal obesity could be an independent risk factor for reflux esophagitis. ${ }^{27}$ In addition, visceral fat may increase the risk of reflux esophagitis through inflammatory cytokines. ${ }^{28}$ Abdominal obesity rather than BMI could thus be a better tool for detecting GERD in Korea, where not many people are severely obese.

The scores of the SSS and ESS, the parameters used to evaluate daytime sleepiness in the current study, were higher in subjects with OSA with GERD than in subjects with OSA without GERD. In addition to the deterioration of sleep quality due to OSA, nocturnal reflux symptoms due to GERD might lead to daytime sleepiness. GERD has also been suggested to be associated with anxiety and depression. ${ }^{29}$ The link between psychological distress, such as anxiety and depression, and GERD is complicated. Gastroesophageal reflux symptoms and psychological distress can affect each other. In other words, gastroesophageal reflux symptoms can aggravate psychological distress, but psychological distress can also provoke them. Furthermore, quality of life in GERD may be more related to anxiety and depression than to symptom severity. ${ }^{12}$ Depression scores were significantly higher in the GERD group than in the non-GERD group, indicating a relation between depression and GERD. In a population-based study, the prevalence of GERD was significantly higher in patients with major depressive disorder, and a multivariate logistic regression analysis showed that major depressive disorders were significantly associated with GERD. ${ }^{30}$ Sleep dysfunction, anxiety, and depression have all been associated with GERD, especially in non-erosive reflux disease (NERD). ${ }^{31,32}$ In fact, anxiety and depression may play an important role in the occurrence of GERD, and especially in NERD. ${ }^{33}$ A Taiwanese study also suggested that GERD increases the risks of depression, anxiety, and sleep disorders. ${ }^{34}$ The evidence of the linkage between depression and OSA is however inconclusive. Some authors have reported that patients with OSA do not show a clinically significant degree of depression or have levels of depressive symptoms no higher than healthy controls. ${ }^{35}$ Other authors have however found that OSA is associated with clinically significant depression. ${ }^{36}$ Depression may also play an important role in non-adherence, with a recent metaanalysis finding that depression is associated with poor compliance to medications across a range of chronic conditions. ${ }^{37}$ One postal survey of 178 established continuous positive airway pressure (CPAP) users found an association between depression scores and non-compliance to therapy. ${ }^{38}$ In a recent study of 240 CPAP-naïve patients with OSA, depression was independently associated with poorer adherence during home-based auto-titrating continuous positive airway pressure therapy. ${ }^{39}$ Poor adherence to CPAP therapy remains the greatest obstacle to the treatment of OSA. In patients with OSA with GERD, a high depression score can be a risk factor for non-compliance with CPAP adherence. Treatment of GERD in patients with OSA may thus be a potential target for clinicians to enhance adherence to CPAP therapy.

The strengths of the present study are as follows. First, we determined the prevalence of GERD in patients with OSA. Second, we tried to find out if there is a difference in the prevalence of GERD according to the severity of OSA patients. Third, it was suggested that there would be a relationship between nocturnal reflux symptoms and obesity related parameters. Finally, it was revealed that GERD and depression might be related in patients with OSA patients.

The current study has several limitations. Firstly, this was a retrospective analysis, and the results might have been affected by the biases and limitations of such studies. Secondly, the number of patients with GERD among the subjects who underwent PSG was small, which obviously limits the interpretation of the results. Thirdly, because we did not conduct endoscopy, we could not compare patients with GERD to other subgroups such as those with ERD and NERD. However, as endoscopy is not required for a GERD diagnosis, we used validated questionnaires to diagnose GERD and performed PSG for the OSA diagnosis. Finally, some of these persons who might be classified as non-GERD had real GERD. In this study, we could not to clarify the causative relationship between OSA and GERD. A prospective study is required on a large scale to verify the cause-and-effect relationship. Finally, some OSA patients with nocturnal reflux symptoms did not fulfill the definition of GERD of this study and these persons who were 
classified as non-GERD might have possibility of real GERD.

Taken together, the present study does not show a relationship between the severity of OSA and the presence of GERD in a sample of patients. The presence of GERD and the severity of OSA did not correlate either. However, OSA and GERD seem to be linked indirectly, as we found 2 parameters of daytime sleepiness to be associated with GERD. Nocturnal gastroesophageal reflux symptoms seem to be related to obesity in OSA.

Acknowledgements: We would like to thank staffs of the sleep clinic of St. Paul's Hospital, The Catholic University of Korea for helping to collect the questionnaires during overnight polysomnography.

\section{Financial support: None.}

\section{Conflicts of interest: None.}

Author contributions: Hyeon Hui Kang collected patients, analyzed the data, drafted the manuscript, tables, and figures, and approved the final version to be published; Chul-Hyun Lim analyzed the data, revised the manuscript, tables, and figures, and approved the final version; Jung Hwan Oh designed the study, analyzed the data, revised the draft, and approved the final version; Min-Jae Cho helped to analyze data, and approved the final version; and Sang Haak Lee collected patients and approved the final version.

\section{References}

1. Jung HK. Epidemiology of gastroesophageal reflux disease in Asia: a systematic review. J Neurogastroenterol Motil 2011;17:14-27.

2. Oh JH. Gastroesophageal reflux disease: recent advances and its association with sleep. Ann N Y Acad Sci 2016;1380:195-203.

3. Bortolotti M, Gentilini L, Morselli C, Giovannini M. Obstructive sleep apnoea is improved by a prolonged treatment of gastrooesophageal reflux with omeprazole. Dig Liver Dis 2006;38:78-81.

4. Regenbogen E, Helkin A, Georgopoulos R, Vasu T, Shroyer AL. Esophageal reflux disease proton pump inhibitor therapy impact on sleep disturbance: a systematic review. Otolaryngol Head Neck Surg 2012;146:524-532.

5. Leggett CL, Gorospe EC, Calvin AD, et al. Obstructive sleep apnea is a risk factor for Barrett's esophagus. Clin Gastroenterol Hepatol 2014;12:583-588, e1.

6. Mannarino MR, Di Filippo F, Pirro M. Obstructive sleep apnea syndrome. Eur J Intern Med 2012;23:586-593.

7. OECD. OECD health statistics 2020. Available from URL: http:// www.oecd.org/health/health-data.htm (accessed 23 March, 2021).

8. Araghi MH, Chen YF, Jagielski A, et al. Effectiveness of lifestyle interventions on obstructive sleep apnea (OSA): systematic review and meta- analysis. Sleep 2013;36:1553-1562, 1562A-1562E.

9. Sleep-related breathing disorders in adults: recommendations for syndrome definition and measurement techniques in clinical research. The report of an American academy of sleep medicine task force. Sleep 1999;22:667-689.

10. Cho YS, Choi MG, Jeong JJ, et al. Prevalence and clinical spectrum of gastroesophageal reflux: a population-based study in Asan-si, Korea. Am J Gastroenterol 2005;100:747-753.

11. Jeong JJ, Choi MG, Cho YS, et al. Chronic gastrointestinal symptoms and quality of life in the Korean population. World J Gastroenterol 2008;14:6388-6394.

12. Oh JH, Kim TS, Choi MG, et al. Relationship between psychological factors and quality of life in subtypes of gastroesophageal reflux disease. Gut Liver 2009;3:259-265.

13. Oh JH, Choi MG, Park JM, et al. The clinical characteristics of gastroesophageal reflux disease in patients with laryngeal symptoms who are referred to gastroenterology. Dis Esophagus 2013;26:465-469.

14. You CR, Oh JH, Seo M, et al. Association between non-erosive reflux disease and high risk of obstructive sleep apnea in Korean population. J Neurogastroenterol Motil 2014;20:197-204.

15. Zigmond AS, Snaith RP. The hospital anxiety and depression scale. Acta Psychiatr Scand 1983;67:361-370.

16. Oh SM, Min KJ, Park DB. A study on the standardization of the hospital anxiety and depression scale for Koreans; a comparison of normal, depressed, and anxious groups. J Korean Neuropsychiatr Assoc 1999;38:289-296.

17. Hoddes E, Zarcone V, Smythe H, Phillips R, Dement WC. Quantification of sleepiness: a new approach. Psychophysiology 1973;10:431-436.

18. Johns MW. A new method for measuring daytime sleepiness: the Epworth sleepiness scale. Sleep 1991;14:540-545.

19. Cho YW, Lee JH, Son HK, Lee SH, Shin C, Johns MW. The reliability and validity of the Korean version of the Epworth sleepiness scale. Sleep Breath 2011;15:377-384.

20. Ashwell M, Gunn P, Gibson S. Waist-to-height ratio is a better screening tool than waist circumference and BMI for adult cardiometabolic risk factors: systematic review and meta-analysis. Obes Rev 2012;13:275-286.

21. Kaneko R, Nakazaki N, Tagawa T, et al. A new index of abdominal obesity which effectively predicts risk of colon tumor development in female Japanese. Asian Pac J Cancer Prev 2014;15:1005-1010.

22. Kim HN, Vorona RD, Winn MP, Doviak M, Johnson DA, Ware JC. Symptoms of gastro-oesophageal reflux disease and the severity of obstructive sleep apnoea syndrome are not related in sleep disorders center patients. Aliment Pharmacol Ther 2005;21:1127-1133.

23. Morse CA, Quan SF, Mays MZ, Green C, Stephen G, Fass R. Is there a relationship between obstructive sleep apnea and gastroesophageal reflux disease? Clin Gastroenterol Hepatol 2004;2:761-768.

24. Kim Y, Lee YJ, Park JS, et al. Associations between obstructive sleep apnea severity and endoscopically proven gastroesophageal reflux disease. Sleep Breath 2018;22:85-90.

25. Ju G, Yoon IY, Lee SD, Kim N. Relationships between sleep disturbances and gastroesophageal reflux disease in Asian sleep clinic referrals. J Psychosom Res 2013;75:551-555. 
26. Basoglu OK, Vardar R, Tasbakan MS, et al. Obstructive sleep apnea syndrome and gastroesophageal reflux disease: the importance of obesity and gender. Sleep Breath 2015;19:585-592.

27. Kang MS, Park DI, Oh SY, et al. Abdominal obesity is an independent risk factor for erosive esophagitis in a Korean population. J Gastroenterol Hepatol 2007;22:1656-1661.

28. Nam SY, Choi IJ, Ryu HK, et al. The effect of abdominal visceral fat, circulating inflammatory cytokines, and leptin levels on reflux esophagitis. J Neurogastroenterol Motil 2015;21:247-254.

29. Sifrim D, Zerbib F. Diagnosis and management of patients with reflux symptoms refractory to proton pump inhibitors. Gut 2012;61:13401354.

30. Chou PH, Lin CC, Lin CH, et al. Prevalence of gastroesophageal reflux disease in major depressive disorder: a population-based study. Psychosomatics 2014;55:155-162.

31. Kim JY, Kim N, Seo PJ, et al. Association of sleep dysfunction and emotional status with gastroesophageal reflux disease in Korea. J Neurogastroenterol Motil 2013;19:344-354.

32. Choi JM, Yang JI, Kang SJ, et al. Association between anxiety and depression and gastroesophageal reflux disease: results from a large cross- sectional study. J Neurogastroenterol Motil 2018;24:593-602.

33. Yang XJ, Jiang HM, Hou XH, Song J. Anxiety and depression in patients with gastroesophageal reflux disease and their effect on quality of life. World J Gastroenterol 2015;21:4302-4309.

34. You ZH, Perng CL, Hu LY, et al. Risk of psychiatric disorders following gastroesophageal reflux disease: a nationwide population-based cohort study. Eur J Intern Med 2015;26:534-539.

35. Reynolds CF 3rd, Kupfer DJ, McEachran AB, Taska LS, Sewitch DE, Coble PA. Depressive psychopathology in male sleep apneics. J Clin Psychiatry 1984;45:287-290.

36. Hobzova M, Prasko J, Vanek J, et al. Depression and obstructive sleep apnea. Neuro Endocrinol Lett 2017;38:343-352.

37. Grenard JL, Munjas BA, Adams JL, et al. Depression and medication adherence in the treatment of chronic diseases in the United States: a meta-analysis. J Gen Intern Med 2011;26:1175-1182.

38. Kjelsberg FN, Ruud EA, Stavem K. Predictors of symptoms of anxiety and depression in obstructive sleep apnea. Sleep Med 2005;6:341-346.

39. Law M, Naughton M, Ho S, Roebuck T, Dabscheck E. Depression may reduce adherence during CPAP titration trial. J Clin Sleep Med 2014;10:163-169. 